\title{
CHARACTERIZATIONS OF PARACOMPACTNESS AND SUBPARACOMPACTNESS USING STAR REDUCIBILITY
}

\author{
HOWARD H. WICKE AND JOHN M. WORRELL, JR.
}

(Communicated by Dennis Burke)

\begin{abstract}
Paracompactness and subparacompactness are characterized using star reducibility and regular (respectively, subregular) refinability. Applications are made to $p$-spaces, developable spaces, and metrizable spaces.
\end{abstract}

\section{INTRODUCTION}

We define a covering property, called star reducibility, possessed by all $\delta \theta$ refinable, countably subparacompact spaces, and we use it in combination with regular refinability (respectively, subregular refinability) to characterize $T_{2}$ paracompact spaces (respectively, subparacompact spaces). We also obtain characterizations of paracompactness and subparacompactness by strengthening star reducibility to incorporate some aspects of the regular or subregular refinability properties. We apply these results in characterizing paracompact $p$ spaces, subparacompact $p$-spaces, developable spaces and metrizable spaces.

Recall that $\left[\mathrm{Wo}_{1}\right]$ if $\mathscr{H}$ and $\mathscr{H}$ are collections of sets, $\mathscr{K}$ is said to be regularly inscribed in $\mathscr{H}$ at $p \in \bigcup \mathscr{K}$ if and only if, for all $U, V \in \mathscr{K}$, if $p \in U \cap V$, then there exists $W \in \mathscr{H}$ such that $U \cup V \subseteq W$. A space $X$ is called regularly refinable provided that every open cover $\mathscr{U}$ of $X$ has a regular refinement, i.e., an open refinement $\mathscr{V}$ such that $\mathscr{V}$ is regularly inscribed in $\mathscr{U}$ at all $p \in X$. A space $X$ is called subregularly refinable if and only if, for every open cover $\mathscr{U}$ of $X$, there is a sequence $\left\langle\mathscr{V}_{n}: n \in \omega\right\rangle$ of open refinements of $\mathscr{U}$ such that, for each $p \in X$, there is some $n \in \omega$ such that $\mathscr{V}_{n}$ is regularly inscribed in $\mathscr{U}$ at $p$.

The terminology we use concerning refinements and partial refinements can be found in $\left[B_{2}\right]$.

Received by the editors October 30, 1987 and, in revised form, April 17, 1990; some of the results of this paper were presented at the Ninety-Third Annual Meeting of the American Mathematical Society in San Antonio, Texas, January 23, 1987.

1980 Mathematics Subject Classification (1985 Revision). Primary 54D18, 54E18.

Key words and phrases. Paracompactness, subparacompactness, star reducible, regular and subregular star reducibility, regular and subregular refinability. 
We introduce the following concepts:

Definition 1.1. A cover $\mathscr{U}$ of a space $X$ is called regularly rigid if and only if no subcollection of $\mathscr{U}$ of cardinality less than $|\mathscr{U}|$ covers $X$ and $|\mathscr{U}|$ is regular or $1<|\mathscr{U}|<\omega$.

Definition 1.2. A space $X$ is called star reducible if and only if, for every regularly rigid open cover $\mathscr{H}$ of $X$, there exists a sequence $\left\langle\mathscr{G}_{n}: n \in \omega\right\rangle$ of open covers of $X$ such that for all $p \in X$ there are $n \in \omega$ and $\mathscr{H}^{\prime} \subseteq \mathscr{H}$ such that $\left|\mathscr{H}^{\prime}\right|<|\mathscr{H}|$ and $\mathscr{H}^{\prime}$ covers $\operatorname{st}\left(p, \mathscr{G}_{n}\right)$. Such a sequence will be called an (SR)-sequence for $\mathscr{H}$. A space $X$ is called (star reducible) ${ }^{r}$ if and only if every uncountable regularly rigid open cover of $X$ has an (SR)-sequence.

A space $X$ is called subregularly star reducible if and only if, for every regularly rigid open cover $\mathscr{H}$ of $X$, there is an (SR)-sequence $\left\langle\mathscr{G}_{n}: n \in \omega\right\rangle$ for $\mathscr{H}$ such that (i) for all $p \in X$ and $n \in \omega$, there is a $j \in \omega$ such that $n<j$ and $\mathscr{G}_{j}$ is regularly inscribed in $\mathscr{G}_{n}$ at $p$ and (ii) for all $n \in \omega, \mathscr{G}_{n+1}$ refines $\mathscr{G}_{n}$.

A space $X$ is called regularly star reducible if and only if every regularly rigid open cover $\mathscr{H}$ has an (SR)-sequence $\left\langle\mathscr{G}_{n}: n \in \omega\right\rangle$ such that each $\mathscr{G}_{n+1}$ regularly refines $\mathscr{G}_{n}$.

In the sequel, we will use the terminology (SSR)- (respectively, (RSR)-) sequence for $\mathscr{H}$ to designate a sequence $\left\langle\mathscr{G}_{n}: n \in \omega\right\rangle$ satisfying the obviously corresponding condition in Definition 1.2 in relation to $\mathscr{H}$.

Remark 1.3. Regular star reducibility implies subregular star reducibility implies star reducibility implies (star reducibility) ${ }^{r}$.

Remark 1.4. If $X$ is a $\delta \theta$-refinable (= submeta-Lindelöf) countably subparacompact space, or if $X$ is $\theta$-refinable and subnormal (= every finite open cover has a countable closed cover $[\mathrm{K}])$, then $X$ is star reducible.

Proof. If $X$ is $\delta \theta$-refinable, then $X$ is (star reducible) ${ }^{r}$, and Lemma 2.3 shows that countable subparacompactness implies star reducibility for countable covers. The second part follows similarly.

Remark 1.5. Strict $p$-spaces and developable spaces are star reducible.

Proof. This follows from the intrinsic characterization of [BS] in the first case and directly from the definition in the second.

Remark 1.6. The properties defined in Definition 1.2 are closed-hereditary.

Sketch of proof. Suppose a space $X$ has one of the properties and $\mathscr{K}$ is a regularly rigid open cover of a closed subspace $F$ of $X$. For each $K \in \mathscr{K}$, choose an open $D_{K}$ such that $D_{K} \cap F=K$. Then $\left\{D_{K}: K \in \mathscr{K}\right\} \cup\{X \backslash F\}$ (or, possibly, $\left\{D_{K}: K \in \mathscr{K}\right\}$, if $\left.|\mathscr{K}|<\omega\right)$ is a regularly rigid open cover of $X$. Let $\left\langle\mathscr{G}_{n}: n \in \omega\right\rangle$ be the corresponding sequence given by Definition 1.2. Then $\left\langle\mathscr{G}_{n} \mid F: G \in \mathscr{G}_{n}\right\rangle$, where $\mathscr{G}_{n} \uparrow F=\left\{G \cap F: G \in \mathscr{G}_{n}\right\}$, defines the appropriate sequence for $F$. 
Our main theorems are the following:

Theorem 1.7. Let $X$ be a topological space. Then the following are equivalent:

(a) $X$ is subparacompact.

(b) $X$ is countably subparacompact, (star reducible) ${ }^{r}$, and subregularly refinable.

(c) $X$ is star reducible and subregularly refinable.

(d) $X$ is subregularly star reducible.

Theorem 1.8. Let $X$ be a $T_{1}$ topological space. Then the following are equivalent :

(a) $X$ is $T_{2}$ paracompact.

(b) $X$ is countably subparacompact, (star reducible $)^{r}$, and regularly refinable.

(c) $X$ is star reducible and regularly refinable.

(d) $X$ is regularly star reducible.

\section{Proof OF TheOREM 1.7}

We first prove several lemmas, using the following notation and terminology: The set of all subsets of a set $S$ is denoted by $\mathscr{P}(S)$. If we write that $\bigcup\left\{\mathscr{D}_{n}: n \in \omega\right\}$ is a $\sigma$-discrete open (respectively, closed) collection in a space $X$, we intend that each $\mathscr{D}_{n}$ is discrete and open (respectively, closed) in $X$. If $X$ is a space and $A \subseteq B \subseteq X$, then $\mathrm{cl}_{B}(\mathscr{A})$ denotes the closure of $A$ in the subspace topology of $B$; if $B=X$, we also write $\bar{A}$ for this. If $\mathscr{A} \subseteq \mathscr{P}(B)$, we write $\operatorname{cl}_{B}(\mathscr{A})$ for $\left\{\mathrm{cl}_{B}(A): A \in \mathscr{A}\right\}$ and $\overline{\mathscr{A}}$ for $\{\bar{A}: A \in \mathscr{A}\}$. If $X$ is a space and $\mathscr{U}, \mathscr{V} \subseteq \mathscr{P}(X)$, we say that $\mathscr{U}$ is a closurewise partial refinement of $\mathscr{V}$ provided that $\overline{\mathscr{U}}$ partially refines $\mathscr{V}$. If $\mathscr{U}$ is also a cover of $X$, then we say that $\mathscr{U}$ is a closurewise refinement of $\mathscr{V}$.

Lemma 2.1. Let $X$ be a space, let $F \subseteq X$ be closed, and suppose $\mathscr{A}$ is a collection of sets discrete and closed relative to $F$. Then $\mathscr{A}$ is discrete and closed relative to $X$.

Lemma 2.2. Let $\mathscr{A}$ be a closed discrete collection of subsets of a space $X$ and, for each $A \in \mathscr{A}$, let $\mathscr{C}(A)$ be a collection of sets closed and discrete relative to A. Then $\mathscr{F}=\bigcup\{\mathscr{C}(A): A \in \mathscr{A}\}$ is a closed discrete collection relative to $X$.

Proof. Let $x \in X$. By Lemma 2.1, the set

$$
X \backslash(\bigcup\{A \in \mathscr{A}: x \notin A\} \cup \bigcup\{E \in \mathscr{C}(A): x \in A \text { and } x \notin E\})
$$

is a neighborhood of $x$ meeting at most one element of $\mathscr{F}$.

Lemma 2.3. If a space $X$ is countably subparacompact and (star reducible) ${ }^{r}$, then $X$ is star reducible.

Proof. A space $X$ is countably subparacompact if and only if, for every countable open cover $\mathscr{U}$ of $X$, there is a sequence $\left\langle\mathscr{G}_{n}: n \in \omega\right\rangle$ of open covers such 
that for all $x \in X$ there are an $n \in \omega$ and a $U \in \mathscr{U}$ such that $\operatorname{st}\left(x, \mathscr{G}_{n}\right) \subseteq U$ (by [K, Theorem 2.3]). This, together with (star reducibility) ${ }^{r}$, implies that all regularly rigid open covers of $X$ have (SR)-sequences.

Lemma 2.4. Let $X$ be a star reducible space. If $X$ is subregularly refinable (respectively, regularly refinable), then $X$ is subregularly star reducible (respectively, regularly star reducible).

Proof. Let $X$ be a star reducible, subregularly refinable space, and suppose $\mathscr{H}$ is a regularly rigid open cover of $X$. Then there is an (SR)-sequence $\left\langle\mathscr{G}_{n} \in \omega\right\rangle$ for $\mathscr{H}$. Let $\mathscr{G}_{0}^{\prime}=\mathscr{G}_{0}$ and let $\left\langle\mathscr{V}_{0 m}: m \in \omega\right\rangle$ be a subregular refining sequence for $\mathscr{G}_{0}^{\prime}$. Let $\mathscr{G}_{1}^{\prime}$ be an open refinement of $\mathscr{G}_{0}^{\prime}, \mathscr{G}_{1}$, and $\mathscr{V}_{00}$. Let $\left\langle\mathscr{V}_{1 m}: m \in \omega\right\rangle$ be a subregular refining sequence of $\mathscr{G}_{1}^{\prime}$, and let $\mathscr{G}_{2}^{\prime}$ be an open refinement of $\mathscr{G}_{1}^{\prime}, \mathscr{G}_{2}, \mathscr{V}_{01}$, and $\mathscr{V}_{10}$. By an obvious induction, we obtain an (SSR)-sequence $\left\langle\mathscr{G}_{n}^{\prime}: n \in \omega\right\rangle$ for $\mathscr{H}$. The proof for the regularly refinable case is similar.

Lemma 2.5. Let $\mathscr{A}$ be a closed-hereditary class of spaces which has a nonsubparacompact member. Let $\kappa$ be the minimum cardinal such that some $X \in \mathscr{A}$ has an open cover $\mathscr{H}$ of cardinality $\kappa$ which has no $\sigma$-discrete closed refinement. Then if $\kappa \geq \omega$, it is regular.

Proof. We may assume $\kappa>\omega$. Let $X$ and $\mathscr{H}$ be as described, and let $\mathscr{H}$ be well-ordered in type $\kappa$. Let $\Sigma$ denote the collection of proper initial segments of $\mathscr{H}$ relative to this well-ordering, and let $\prec$ denote the well-ordering of $\Sigma$ determined by proper set inclusion. Suppose $\lambda=\operatorname{cf}(\kappa)<\kappa$, and let $\Lambda$ be a $\prec$-cofinal subset of $\Sigma$ of cardinality $\lambda$. Then $\{\bigcup \tau: \tau \in \Lambda\}$ is an open cover of $X$ which has a $\sigma$-discrete closed refinement $\mathscr{D}=\bigcup\left\{\mathscr{D}_{n}: n \in \omega\right\}$. Fix $n \in \omega$ and $D \in \mathscr{D}_{n}$. Let $\tau_{D}=\prec-\min \{\zeta \in \Lambda: D \subseteq \bigcup \zeta\}$. Since $\left|\tau_{D}\right|<\kappa$ and $D \in \mathscr{A}$, it follows from the definition of $\kappa$ that the relatively open cover $\left\{D \cap U: U \in \tau_{D}\right\}$ of $D$ has a $\sigma$-discrete closed refinement $\bigcup\left\{\mathscr{C}(D)_{n}: n \in \omega\right\}$ relative to $D$. By Lemmas 2.1 and 2.2, each of sets $\mathscr{F}_{n m}=\bigcup\left\{\mathscr{C}(D)_{m}: D \in \mathscr{D}_{n}\right\}$ is a closed discrete collection relative to $X$. But then $\bigcup\left\{\mathscr{F}_{n m}:\langle n, m\rangle \in \omega \times \omega\right\}$ is a $\sigma$-discrete closed refinement of $\mathscr{H}$, which is a contradiction. Hence $\kappa$ is regular.

Lemma 2.6. Let $\mathscr{M}$ be a closed-hereditary class of $T_{3}$-spaces which has a nonparacompact member. Let $\kappa$ be the minimum cardinal such that some $X \in \mathscr{M}$ has an open cover $\mathscr{H}$ of cardinality $\kappa$ which has no closurewise $\sigma$-discrete open refinement. Then if $\kappa \geq \omega$, it is regular.

Proof. Assume $\kappa>\omega$, let $X$ and $\mathscr{H}$ be as described, and define $\prec, \Sigma, \lambda$, and $\Lambda$ as in the proof of Lemma 2.5. Then $\{\bigcup \tau: \tau \in \Lambda\}$ is an open cover of $X$ which has a closurewise $\sigma$-discrete open refinement $\bigcup\left\{\mathscr{D}_{n}: n \in \omega\right\}$. Fix $n \in \omega$ and $D \in \mathscr{D}_{n}$. Let $\tau_{D}=\prec-\min \{\zeta \in \Sigma: \bar{D} \subseteq \bigcup \zeta\}$. Since $\left|\tau_{D}\right|<\kappa$ and $\bar{D} \in \mathscr{M}$, there is, relative to $\bar{D}$, a closurewise $\sigma$-discrete open refinement $\bigcup\left\{\mathscr{E}(D)_{m}: m \in \omega\right\}$ of $\left\{\bar{D} \cap V: V \in \tau_{D}\right\}$. Fix $m \in \omega$. If $E \in \mathscr{E}(D)_{m}$, choose $G_{E} \subseteq \bigcup \tau_{D}$ open in $X$ such that $E=\bar{D} \cap G_{E}$. Also choose $V_{E} \in \tau_{D}$ such that 
$\operatorname{cl}_{\bar{D}}(E) \subseteq V_{E}$; then $\overline{D \cap G_{E}} \subseteq V_{E}$. From this and Lemma 2.1 it follows that $\operatorname{cl}_{\bar{D}}\left(\mathscr{E}(D)_{m}\right)$ is discrete and closed in $\bar{D}$, hence in $X$, and partially refines $\mathscr{H}$. By Lemma 2.2, $\bigcup\left\{\operatorname{cl}_{\bar{D}}\left(\mathscr{E}(D)_{m}\right): D \in \mathscr{D}_{n}\right\}$ is closed and discrete in $X$. Thus, if $\mathscr{C}(D)_{m}=\left\{D \cap G_{E}: E \in \mathscr{C}_{m}(D)\right\}$, then $\mathscr{F}_{n m}=\bigcup\left\{\mathscr{C}(D)_{m}: D \in \mathscr{D}_{n}\right\}$ is open and discrete in $X$. From this it follows readily that $\bigcup\left\{\mathscr{F}_{n m}:\langle n, m\rangle \in \omega \times \omega\right\}$ is a closurewise $\sigma$-discrete open refinement of $\mathscr{H}$. Since this is a contradiction of the definition of $\kappa$, it follows that $\kappa$ is regular.

Proof of Theorem 1.7. (a) $\Rightarrow$ (b). Let $X$ be a subparacompact space, and let $\mathscr{U}$ be an open cover of $X$. By [B2, Theorem 3.1(v)] there is a sequence $\left\langle\mathscr{G}_{n}: n \in \omega\right\rangle$ of open refinements of $\mathscr{U}$ such that, for all $p \in X$, there is $n \in \omega$ such that $\operatorname{st}\left(p, \mathscr{G}_{n}\right) \subseteq U$ for some $U \in \mathscr{U}$. It follows from the definitions that $X$ satisfies (b).

(b) $\Rightarrow$ (c). By Lemma 2.3.

(c) $\Rightarrow$ (d). By Lemma 2.4 .

(d) $\Rightarrow(a)$. If this is not true, then there exists a smallest cardinal $\kappa$ such that there exist a nonsubparacompact subregularly reducible space $X$ and an open cover $\mathscr{H}$ of $X$ of cardinality $\kappa$ which does not have a $\sigma$-discrete closed refinement. By Lemma 2.5, if $\kappa$ is not finite it is regular. Thus $\mathscr{H}$ is regularly rigid. Hence there is an (SSR)-sequence $\left\langle\mathscr{G}_{n}: n \in \omega\right\rangle$ for $\mathscr{H}$.

Case 1. $\kappa \geq \omega$. Assume that $\mathscr{H}$ is well-ordered in type $\kappa$. Let $\Sigma$ be the collection of initial segments of $\mathscr{H}$ under this well-ordering and let $\prec$ denote the well-ordering of $\Sigma$ determined by proper set inclusion. Since $\kappa$ is regular, for each $p \in X$ there is $n \in \omega$ and $\zeta \in \Sigma$ such that $\operatorname{st}\left(p, \mathscr{G}_{n}\right) \subseteq \bigcup \zeta$. Let $\tau(p)$ denote the $\prec$-least such $\zeta$ and let $n(p)$ denote the least $n \in \omega$ such that $\tau(p)$ covers $\operatorname{st}\left(p, \mathscr{G}_{n}\right)$. For each $p \in X$, let $k(p)=\min \{j \in \omega: j>n(p)$, and $\mathscr{G}_{j}$ is regularly inscribed in $\mathscr{G}_{n(p)}$ at $\left.p\right\}$. Define for all $\langle r, s\rangle \in \omega \times \omega$, $M(r, s)=\{p \in X: r=n(p)$ and $s=k(p)\}$. Then $X=\bigcup\{M(r, s):\langle r, s\rangle \in$ $\omega \times \omega\}$. For all $\xi \in \Sigma$, let $M(r, s, \xi)=\{p \in M(r, s): \xi=\tau(p)\}$. Suppose $\zeta, \xi \in \Sigma$, and $\zeta \prec \xi$, and $p \in X$ is such that $p \in \overline{M(r, s, \zeta)} \cap \overline{M(r, s, \xi)}$. There is a $G \in \mathscr{G}_{r+s}$ which contains $p$. Hence there exist $q \in G \cap M(r, s, \zeta)$ and $q^{\prime} \in G \cap M(r, s, \xi)$. Since $\zeta \prec \xi=\tau\left(q^{\prime}\right)$, st $\left(q^{\prime}, \mathscr{G}_{r+s}\right)$ is not covered by $\zeta$, so it follows that there is $H \in \mathscr{G}_{r+s}$ such that $q^{\prime} \in H$ and $H$ is not covered by $\zeta$. Since $q^{\prime} \in G \cup H$ and $\mathscr{G}_{r+s}$ is regularly inscribed in $\mathscr{G}_{r}$ at $q^{\prime}$, it follows that some $W \in \mathscr{G}_{r}$ includes $G \cup H$. But $q \in W$ and $\operatorname{st}\left(q, \mathscr{G}_{r}\right) \subseteq \bigcup \zeta$ implies $H \subseteq \bigcup \zeta$, which contradicts the choice of $H$.

From this it follows that $\mathscr{D}(r, s)=\{\overline{M(r, s, \tau)}: \tau \in \Sigma\}$ is a discrete closed collection, and thus $\mathscr{D}=\{\mathscr{D}(r, s):\langle r, s\rangle \in \omega \times \omega\}$ is a $\sigma$-discrete closed cover of $X$. Fix $r, s \in \omega$. If $D \in \mathscr{D}(r, s)$, let $\tau_{D}$ be the element $\zeta \in \Sigma$ such that $D=\overline{M(r, s, \zeta)}$. If $p \in D$, then there exists $G \in \mathscr{G}_{r+s}$ containing $p$ so that $G \cap M\left(r, s, \tau_{D}\right) \neq \varnothing$. Hence $p \in \bigcup \tau_{D}$, so that $D \subseteq \bigcup \tau_{D}$. Because $D$ is closed, it is subregularly star reducible (Remark 1.6). As a result, because $\left|\tau_{D}\right|<\kappa$, there is a $\sigma$-discrete closed (in $D$ ) refinement $\mathscr{C}(D)=$ $\bigcup\left\{\mathscr{C}(D)_{m}: m \in \omega\right\}$ of $\left\{D \cup U: U \in \tau_{D}\right\}$. By Lemmas 2.1 and 2.2, each 
of the sets $\mathscr{F}_{r s m}=\bigcup\left\{\mathscr{C}(D)_{m}: D \in \mathscr{D}(r, s)\right\}$ is a closed and discrete collection of subsets of $X$. It follows that $\bigcup\left\{\mathscr{F}_{r s m}:\langle r, s, m\rangle \in \omega \times \omega \times \omega\right\}$ is a $\sigma$-discrete closed refinement of $\mathscr{H}$. Since $\mathscr{H}$ was assumed to have no such refinement, this involves a contradiction.

Case 2. $\kappa \in \omega$. Consider first $\kappa=2$. Suppose $\mathscr{H}=\left\{V_{0}, V_{1}\right\}$. Let $\Sigma=\mathscr{H}$ be well-ordered by $V_{0} \prec V_{1}$. Let $\left\langle\mathscr{G}_{n}: n \in \omega\right\rangle$ be an (SSR)-sequence for $\mathscr{H}$. For each $p \in X$, there are an $n \in \omega$ and a $V_{i} \in \mathscr{H}$ such that $\operatorname{st}\left(p, \mathscr{G}_{n}\right) \subseteq V_{i}$. Let $\tau(p), k(p)$, and $M(r, s)$ be defined as before and $M(r, s, i)=\{p \in$ $M(r, s): i=\tau(p)\}$. Then, as in Case 1 , each $\mathscr{D}(r, s)$ is a discrete closed collection, but here these collections refine $\left\{V_{0}\right\}$ or $\left\{V_{1}\right\}$, so that $\mathscr{H}$ has a $\sigma$-discrete closed refinement, which is a contradiction. Suppose $\kappa=n>2$, and $\mathscr{H}=\left\{U_{0}, U_{1}, \ldots, U_{n}\right\}$. Let $V_{0}=\bigcup\left\{U_{i}: i \in n\right\}$ and $V_{1}=U_{n}$. Then repeat the above reasoning to get a $\sigma$-discrete closed refinement $\mathscr{F}$ of $\left\{V_{0}, V_{1}\right\}$. The members of $\mathscr{F}$ included in $V_{0}$ are covered by $\left\{U_{0}, \ldots, U_{n-1}\right\}$. Since $n-1<\kappa$, the argument of the last paragraph produces a $\sigma$-discrete closed refinement of $\mathscr{H}$, again yielding a contradiction.

\section{PROOF OF THEOREM 1.8}

Lemma 3.1. If $X$ is a $T_{1}$ regularly star reducible space, then $X$ is $T_{3}$.

Proof. Let $p \in X \backslash F$, where $F$ is closed in $X$. The open cover $\mathscr{H}=\{X \backslash\{p\}$, $X \backslash F\}$ is regularly rigid. Let $\left\langle\mathscr{G}_{n}: n \in \omega\right\rangle$ be an (RSR)-sequence for $\mathscr{H}$. Then there exists $n \in \omega$ such that $\operatorname{st}\left(p, \mathscr{G}_{n}\right) \subseteq X \backslash F$. It follows that $\operatorname{st}\left(p, \mathscr{G}_{n+1}\right)$ and st $\left(F, \mathscr{G}_{n+1}\right)$ separate $p$ and $F$.

Lemma 3.2. If $X$ is a $T_{3}$-space, then $X$ is paracompact if and only if every open cover of $X$ has a closurewise $\sigma$-discrete open refinement.

Proof. If $X$ is $T_{3}$, then every open cover of $X$ has a closurewise open refinement. If $X$ is $T_{2}$ paracompact, [E, Theorem 5.1.12(iv)] implies that every open cover of $X$ has a $\sigma$-discrete open refinement. Sufficiency follows from the cited theorem from [E].

Proof of Theorem 1.8. (a) $\Rightarrow$ (b). Let $X$ be a $T_{2}$ paracompact space. Then $X$ is fully normal [S; E, Theorem 5.1.12(iii)]. Clearly, full normality implies (b).

(b) $\Rightarrow$ (c). By Lemma 2.3.

(c) $\Rightarrow(d)$. By Lemma 2.4.

(d) $\Rightarrow(\mathrm{a})$. If this is not true then, using Lemmas 3.1 and 3.2, there exists a smallest cardinal $\kappa$ such that there exist a $T_{3}$ nonparacompact regularly star reducible space $X$ and an open cover $\mathscr{H}$ of $X$ of cardinality $\kappa$ having no closurewise $\sigma$-discrete open refinement. By Lemma 2.6 , if $\kappa$ is not finite, it is regular. Thus $\mathscr{H}$ is regularly rigid. Hence there is an (RSR)-sequence $\left\langle\mathscr{G}_{n}: n \in \omega\right\rangle$ for $\mathscr{H}$.

Case 1. $\kappa \geq \omega$. Define $\prec, \Sigma$, and $\tau(p)$ as in the proof of Theorem 1.7, Case 1. For all $p \in X$, define $n(p)$ as $\min \left\{m \in \omega: \operatorname{st}\left(p, \mathscr{G}_{m}\right) \subseteq \bigcup \tau(p)\right\}$. 
For each $r \in \omega$ and $\zeta \in \Sigma$, let $E(r, \zeta)=\{p \in X: r=n(p)$ and $\zeta=\tau(p)\}$. Let $S(r, \zeta)=\bigcup\left\{\operatorname{st}\left(p, \mathscr{G}_{r+2}\right): p \in E(r, \zeta)\right\}$. Suppose that $G \in \mathscr{G}_{r+2}, x \in G \cap$ $S(r, \zeta), y \in G \cap S(r, \xi)$, and $\zeta \preceq \xi$. Then there exist $u \in E(r, \zeta), v \in$ $E(r, \xi)$, and $U_{2}, V_{2} \in \mathscr{G}_{r+2}$ such that $\{x, u\} \subseteq U_{2}$ and $\{y, v\} \subseteq V_{2}$. Let $z \in \operatorname{st}\left(v, \mathscr{G}_{r+2}\right)$. Then there exists $W_{2} \in \mathscr{G}_{r+2}$ such that $\{v, z\} \subseteq W_{2}$. By the regular refinability property, there exist $U_{1}, V_{1} \in \mathscr{G}_{r+1}$ such that $U_{2} \cup G \subseteq U_{1}$ and $V_{2} \cup W_{2} \subseteq V_{1}$. Since $y \in G \cap V_{2}$ implies that $y \in U_{1} \cap V_{1}$, there is a $V_{0} \in \mathscr{G}_{r}$ such that $U_{1} \cup V_{1} \subseteq V_{0}$. Hence $\{u, z\} \subseteq U_{0}$. Thus $\operatorname{st}\left(v, \mathscr{G}_{r+2}\right) \subseteq$ st $\left(u, \mathscr{G}_{r}\right) \subseteq \bigcup \zeta$. This implies that $\zeta=\xi$, since $v \in E(r, \xi)$. Hence each $p \in X$ has a neighborhood (in $\mathscr{G}_{r+2}$ ) meeting at most one of the sets $S(r, \zeta$ ) for fixed $r \in \omega$. The above argument also shows that if $p \in \overline{S(r, \zeta)}$, there is $u \in E(r, \zeta)$ such that $p \in \operatorname{st}\left(u, \mathscr{G}_{r}\right) \subseteq \bigcup \zeta$. Thus each $\mathscr{D}_{r}=\{S(r, \zeta): \zeta \in \Sigma\}$ is an open discrete collection and $\bigcup\left\{\mathscr{D}_{r}: r \in \omega\right\}$ is a closurewise $\sigma$-discrete open refinement of $\{\bigcup \zeta: \zeta \in \Sigma\}$. Now we can proceed as in the proof of Lemma 2.6 , starting with the second sentence, in which we replace $\Lambda$ by $\Sigma$ and use the $\mathscr{D}_{r}$ 's as just defined. Then we use the fact that each $\overline{S(r, \zeta)}$ is regularly star reducible (Remark 1.6), to obtain a closurewise $\sigma$-discrete open refinement of $\mathscr{H}$. This involves a contradiction.

Case 2. $\kappa \in \omega$. This is done in a manner analogous to that used in the proof of Theorem 1.7, Case 2.

\section{APPLiCATIONS to $p$-SPACES: EXAMPLES AND ISOCOMPACTNESS}

Recall that a space $X$ is called a monotonic $q$-space (equivalently, $\beta_{c}$-space [W]) if and only if $X$ has a sequence $\left\langle\mathscr{G}_{n}: n \in \omega\right\rangle$ of bases such that, if $x \in X$, for all $n \in \omega, x \in G_{n+1} \subseteq G_{n} \in \mathscr{G}_{n}$ and $y_{n} \in G_{n}$, then $\left\langle y_{n}: n \in \omega\right\rangle$ has a cluster point. Every $p$-space is a monotonic $q$-space and every Tychonoff $\theta$ refinable monotonic $q$-space is a $p$-space $\left[\mathrm{WW}_{1}\right]$. From this and Theorems 1.7 and 1.8 we obtain the following theorem:

Theorem 4.1. For a Tychonoff space $X$, the following are equivalent :

(a) $X$ is a subparacompact (paracompact) p-space.

(b) $X$ is a star reducible subregularly (regularly) refinable monotonic $q$ space.

(c) $X$ is a subregularly (regularly) star reducible p-space.

Recall that a $T_{2}$ paracompact space is metrizable if and only if it has a base of countable order [A], and that a space is developable if and only if it is essentially $T_{1}$, has a base of countable order, and is $\theta$-refinable [WoW]. From these results and 1.7 and 1.8 we obtain the following theorems:

Theorem 4.2. $A T_{1}$-space is developable if and only if it has a base of countable order and is subregularly star reducible.

Theorem 4.3. $A T_{1}$-space is metrizable if and only if it is regularly refinable and star reducible and has a base of countable order. 
Theorem 4.4. $A T_{1}$-space having a base of countable order is metrizable if and only if it is regularly star reducible.

Theorem 4.4 is an analogue of the theorem of [AU] (stated in this paper's terminology): A $T_{2}$-space is metrizable if and only if it has a development $\left\langle\mathscr{G}_{n}: n \in \omega\right\rangle$ such that each $\mathscr{G}_{n+1}$ regularly refines $\mathscr{G}_{n}$. Theorem $4.1(\mathrm{c})$ is an analogue of a characterization of paracompact $p$-spaces obtained by Worrell $\left[\mathrm{Wo}_{2}\right]$ and independently by Davis $[\mathrm{D}]$.

Example 4.5. The space $\omega_{1}$ with the order topology is a $T_{2}$ collectionwise normal regularly refinable space which is not star reducible. This follows from the isocompactness of star reducible spaces (see Remark 4.7).

Example 4.6. The strict $p$-space that is not subparacompact in [B1, Example 4.1] is an example of a metacompact star reducible space (see Remark 1.5) which is not subregularly refinable since it is not subregularly star reducible.

Remark 4.7. The following property implies isocompactness and is obviously weaker than star reducibility.

Definition 4.8. A space $X$ is called weakly star reducible if and only if, for every uncountable regularly rigid open cover $\mathscr{U}$ of $X$, there is a collection $\Xi$ of collections of subsets of $X$ such that:

(1) $|\Xi|<|\mathscr{U}|$,

(2) For all $\mathscr{G} \in \Xi$ and for all $G \in \mathscr{G}, G$ is open in $\bigcup \mathscr{G}$, and

(3) For all $p \in X$, there is $\mathscr{G} \in \Xi$ and $\mathscr{U}^{\prime} \subseteq \mathscr{U}$ such that $\left|\mathscr{U}^{\prime}\right|<|\mathscr{U}|$ and $\operatorname{st}(p, \mathscr{G}) \subseteq \bigcup \mathscr{U}^{\prime}$.

\section{REFERENCES}

[AU] P. S. Alexandroff and P. Urysohn, Une condition nécessaire et suffisante pour qu'une classe $(\mathscr{L})$ soit une classe $(\mathscr{D})$, C. R. Acad. Sci Paris 177 (1923), 1274-1276.

[A] A. V. Arhangel'skiï, Some metrization theorems, Uspekhi Mat. Nauk 18 (1963), 139-145. (Russian)

[B1] D. K. Burke, On subparacompact spaces, Proc. Amer. Math. Soc. 23 (1969), 655-663.

[B2] _ Covering properties, Handbook of Set-Theoretic Topology (K. Kunen and J. E. Vaughan, eds.), North-Holland, Amsterdam, 1984, pp. 349-422.

[BS] D. K. Burke and R. A. Stoltenberg, A note on p-spaces and Moore spaces, Pacific. J. Math. 30 (1969), 601-608.

[D] S. W. Davis, The strict p-space problem, Topology Proc. 10 (1985), 277-292.

[E] R. Engelking, General topology, Polish Scientific Publishers, Warsaw, 1977.

[K] T. R. Kramer, A note on countably subparacompact spaces, Pacific J. Math. 46 (1973), 209-213.

[S] A. H. Stone, Paracompactness and product spaces, Bull. Amer. Math. Soc. 54 (1948), $977-$ 982.

[W] H. H. Wicke, Open continuous images of certain kinds of $M$-spaces and completeness of mappings and spaces, General Topology Appl. 1 (1970), 85-100.

[WW1] H. H. Wicke and J. M. Worrell, Jr., The concept of a $\theta$-refinable embedding, General Topology Appl. 6 (1976), 167-181. 
[WW2] __, Subregular refinablility and subparacompactness, Topology Proc. 10 (1985), 207-220.

[Wo1] J. M. Worrell, Jr., On collections of domains inscribed in a covering in the sense of Alexandroff and Urysohn, Portugal. Math. 26 (1967), 405-420.

[Wo2] _ Concerning the paracompact p-spaces of Arhangel' skil, Notices Amer. Math. Soc. 14 (1967), 949.

[WoW] J. M. Worrell, Jr. and H. H. Wicke, Characterizations of developable topological spaces, Canad. J. Math. 17 (1965), 820-830.

Department of Mathematics, Ohio University, Athens, OHio 45701

1908 W. Wall, Midland, TeXas 79701 